\title{
Towards Multiresolution Phase Retrieval using Electron Ptychography
}

\author{
Charudatta Phatak ${ }^{1}$, Youssef Nashed ${ }^{2}$, Tom Peterka ${ }^{2}$ \\ ${ }^{1}$ Materials Science Division, Argonne National Laboratory, Lemont, IL 60439, USA \\ ${ }^{2}$ Mathematics and Computer Science Division, Argonne National Laboratory, Lemont, IL 60439, \\ USA
}

In conventional transmission electron microscopy imaging, due to the limitations of electromagnetic lenses such as aberrations, and instabilities, the achievable spatial resolution is limited to significantly below the wavelenght of electrons. Furthermore, obtaining quantitative information at the highest spatial resolution is often times difficult. Using Ptychography, it has been shown that it is possible to eliminate the aberrations of electromagnetic lenses and obtain high resolution information beyond that limit of direct imaging [1,2].

The electron-optical phase shift of electrons passing through a thin foil sample in a TEM can be written as a combination of various components arising from the electrostatic potential (mean inner potential, local charge density variations), magnetic vector potential (local magnetization), crystalline lattice potential (atomic structure within the unit cell), and geometric phase (displacement field, strain). All these components of the phase shift typically consist of information at various spatial frequencies that need to be recovered. In this work, we use the extended ptychographical iterative engine (ePIE) to reconstruct the entire complex object wavefunction as well as the complex electron probe wavefunction [3]. The algorithm then iterates between reciprocal and real space and updates both the wavefunctions given the set of experimental constraints until convergence. By varying the mask used in reciprocal space during the reconstruction process, the effective resolution of the recovered phase shift can be controlled allowing a multiresolution recovery from the same data set.

Simulations were performed to test the reconstruction procedure for electron ptychography data. The electron probe was simulated for a typical $C_{s}$ corrected $200 \mathrm{kV}$ JEOL $2100 \mathrm{~F}$ FEG TEM. The diffraction images were then simulated using an input phase and amplitude of the object as a function of scanning the probe along a cartesian grid. Fig. 1(a) shows the simulated defocused electron probe, and (b) shows the corresponding diffraction pattern, and (c) shows the experimental defocused electron probe. The effects of lens aberrations such as astigmatism can be easily seen in the experimental image of the probe. Fig. 2(a) shows the input phase that was used for simulating the diffraction patterns, (b) shows a schematic of probe positions in a ptychographic scan overlaid on the simulated image, and (c) shows the preliminary result of the reconstructed phase shift. We will further discuss the effect of defocus of electron probe, overlap of probe positions during scan and unwrapping of recovered phase. Experimentally, we have implemented the ptychographic acquisition on a $200 \mathrm{kV}$ JEOL 2100F equipped with a spherical aberration corrector and a dedicated Lorentz lens using a Digital Micrograph script. The spatial resolution of this microscope is limited to $0.5 \mathrm{~nm}$ in direct imaging mode. We will discuss the improvement of the spatial resolution particularly in the phase reconstruction performed using ptychography.

References

[1] M. J. Humphry, et. al., Nat. Commun., 3, 730 (2012). 
[2] A. J. D'Alfonso, et. al., Phys. Rev. B, 89, 064101 (2014).

[3] Y. S. G. Nashed et. al., Optics Express, 22, 32082 (2014).

[4] This work was supported by U.S. Department of Energy (DOE), Office of Science, Materials Sciences and Engineering Division.
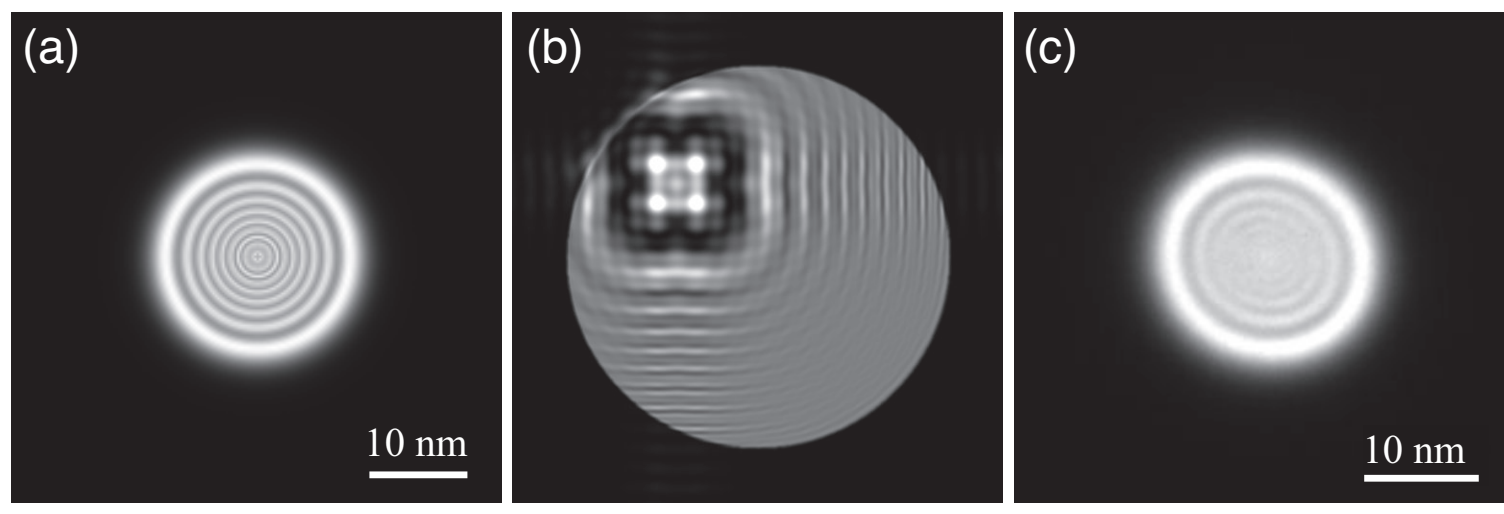

Figure 1: (a) Simulated defocused electron probe for a defocus of $\Delta \mathrm{f}=3 \mu \mathrm{m}$, (b) the corresponding simulated diffraction pattern, and (c) experimental defocused electron probe for a defocus of $\Delta f=2.3$ $\mu \mathrm{m}$.
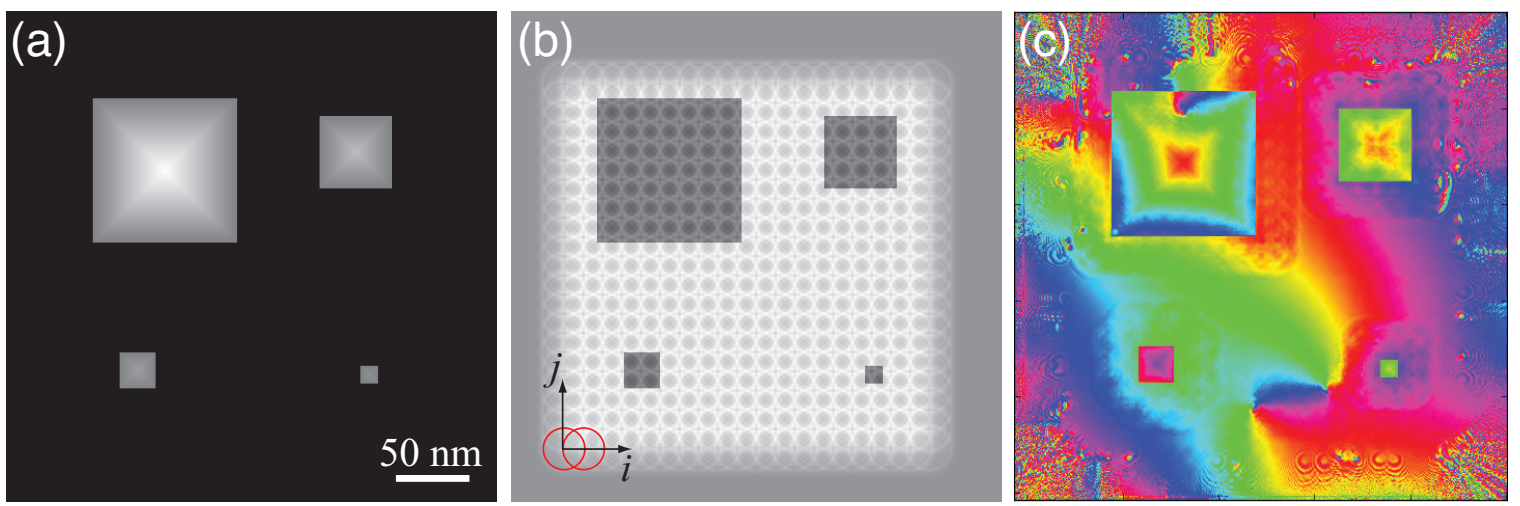

Figure 2: (a) Simulated input phase shift for 4 square magnetic islands showing the closure domain configuration, (b) schematic showing the probe positions during a ptychographic scan overlaid on the infocus image, and (c) the reconstructed phase shift (unwrapped) using ePIE algorithm. 